\title{
Quantitative Analyzing the Spatial Organization of the Organelles in Cancer Cell Using Soft X-Ray Tomography
}

\author{
Jian-Hua Chen ${ }^{1}$, Rosanne Boudreau ${ }^{1}$, Axel Ekman ${ }^{1}$, Gerry McDermott ${ }^{1}$, Mark LeGros ${ }^{1,2}$ and Carolyn \\ Larabel1 $1^{1,2}$ \\ 1. Department of Anatomy, University of California San Francisco, San Francisco, CA, USA \\ 2. Molecular Biophysics \& Integrated Bioimaging, Lawrence Berkeley National Laboratory, Berkeley, \\ CA, USA
}

Using soft x-ray microscopy (SXM), we are able to image and quantitatively analyze the spatial organization of the organelles within cancer cells [1]. Crucial cellular activities, such as cancer invasion and metastasis, mostly take place within a three-dimensional extracellular matrix (ECM) [2]. Thus, the information of three-dimensional organization of organelles in cancer cells is required to tackle questions regarding those highly remodeling activities.

Soft x-ray tomography (SXT), with 50nm spatial resolution [3], provides quantitative information based on the absorption differences from distinct constituents within cells. Image contrast also takes advantage from the property of "water window" of soft x-ray, which means water within cells will be seen as transparent. From the reconstruction tomography, each voxel was given a value, the linear absorption coefficient (LAC), based on the absorption ability. Each organelle has a specific LAC, for example the LAC of lipid droplets is about $0.7 \mu \mathrm{m}^{-1}$, while mitochondria has LAC ranges from 0.31 to $0.35 \mu \mathrm{m}^{-1}$ [4].

The isotropic 3D tomographic image is reconstructed based on projections from different angles. To mimic the cellular growth microenvironment, HT1080, human fibrosarcoma cells, tagged with H2BEGFP and cytoplasmic DsRed2, were cultured within tapered capillaries filled with a reconstructed collagen fiber mixed with growth media, DMEM [2]. The HT1080 cells were first detached from the flask by placing on the ice (4 degree) for 20 minutes. Cell/collagen mixture was loaded into the tip of capillary using a micropipette and then placed in a conventional incubator (37 degree, 5\% $\mathrm{CO}_{2}$ ) for 60 minutes. This enabled us to collect images of cells in a condition that is closer to their $3 \mathrm{D}$ growth environment. Prior to imaging, cells were rapidly frozen by plunging into nitrogen-cooled propane liquid. This not only preserved the cellular structures in their most native state but also protected them from radiation damage.

Our preliminary results have shown that we can quantitatively study the heterogeneity of cell volumes (few hundreds to thousands $\mu \mathrm{m}^{3}$ ) as well as the morphology of the organelles. The volume of HT1080 cells is very diverse due to their rapid morphological remodeling for migration. Based on the LAC histogram, we can discern euchromatin from heterochromatin. To sum up, using soft x-ray microscopy, we are able to quantitatively study the spatial organization of organelles of cancer cells in their most native state [5]. 


\section{References:}

[1] A.A. Ekman et al, Biol Cell 109(1) (2017), p. 24.

[2] K. Wolf et al, J Cell Biol 201(7) (2013), p. 1069.

[3] M.A. Le Gros et al, J Synchrotron Radiat 21(6) (2014), p. 1370.

[4] M. Do et al, Arch Biochem Biophys 581 (2015), p. 111.

[5] The National Center for X-ray Tomography is supported by NIH (P41GM103445) and DOE's Office of Biological and Environmental Research (DE-AC02-5CH11231)
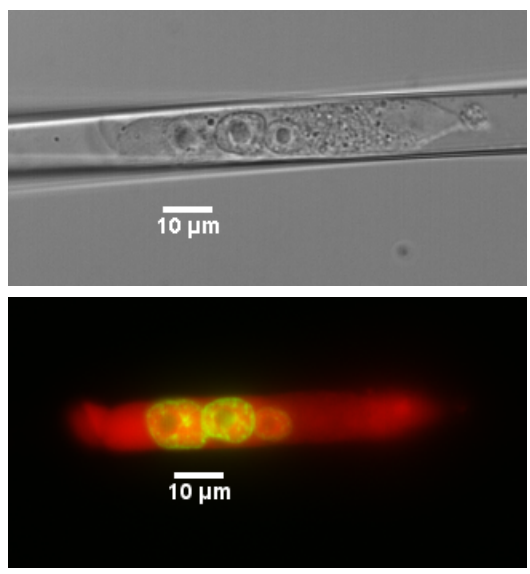

Figure 1. Bright field and fluorescence image of the 3D culture HT1080 cells with reconstructed collagen $(2 \mathrm{mg} / \mathrm{mL})$ in a capillary before freezing down. The cell carries H2B-EGFP and cytoplasmic DsRed2.
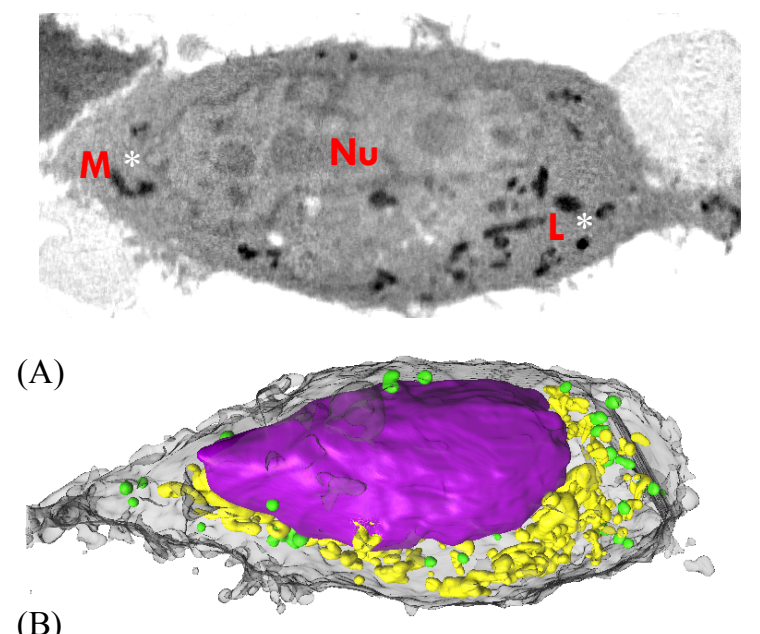

(B)

Figure 2. SXT orthoslice of a human fibrosarcoma cell. (A) An orthoslice from the SXT reconstruction image. Nu: Nucleus, M: Mitochondria, L: Lipids. (B) The 3D visualization and rendering of the same cell. The segmentation is based on the organelle's morphology and LAC value. Purple: Nucleus, Yellow: Mitochondria, Green: Lipids (Scale bar: $2 \mu \mathrm{m}$ ). 